\title{
Reaction cross section calculations for deformed nuclei
}

\author{
J. A. Christley and J. A. Tostevin \\ Department of Physics, School of Physical Sciences, University of Surrey, Guildford, Surrey, GU2 5XH, United Kingdom
}

(Received 11 December 1998)

\begin{abstract}
Optical limit Glauber theory calculations of reaction cross sections, used to deduce nuclear sizes from high energy data, are studied in the case of a deformed projectile (or target). We show that a previously applied formula, used to adjust the root-mean-squared radius deduced assuming spherical projectiles, is consistent with results which treat the projectile deformation explicitly within the reaction calculation. The correct interpretation of this formula in studies of reaction cross sections is clarified. [S0556-2813(99)04304-6]
\end{abstract}

PACS number(s): 21.10.Gv, 11.80.Fv, 25.10.+s, 27.20.+n

The optical limit (OL) approximation to Glauber theory $[1,2]$ has been used frequently in analyses to extract empirical root-mean-squared (rms) matter radii of nuclei from intermediate energy reaction and interaction cross section measurements [3-5]. The inputs to this model are the projectile and target nucleus one-body densities. Their geometric overlap at a given impact parameter, when multiplied by an appropriate nucleon-nucleon $(N N)$ reaction cross section, determines the calculated projectile-target reaction cross section. This is then compared with the measurements. This approach works very well for localized nuclei where nucleons occupy a well-defined volume [3]. For nuclei with weakly bound and delocalized nucleons, recent theoretical analyses [6-8] have shown that projectile excitation and breakup effects are important. Then a more explicit fewbody treatment is necessary for quantitative calculations of the reaction cross sections. This weak binding effect alters (increases) the transparency of the collision at larger impact parameters, reducing the reaction cross section for a given projectile rms size.

Our interest here is the reaction cross section of an assumed localized quadrupole-deformed projectile with deformation parameter $\beta$. At high energy, in the sudden or adiabatic approximation limit, and in a given collision, the deformed projectile nucleus will traverse the target nucleus with a fixed orientation $\hat{\boldsymbol{\Omega}}$ (Fig. 1). The transparency of the collision and cross section for a given $\hat{\mathbf{\Omega}}$ will depend sensitively on this projectile orientation, particularly for neargrazing-impact parameters $b$. The physical cross section, for an assumed unpolarized incident projectile beam, is then the average of such cross sections over all orientations [9].

To date, even when the projectile nuclei are deformed, OL reaction calculations have been carried out for spherical densities, e.g., [10,11]. For nuclei with quadrupole deformation $\beta$, the effects of deformation were then discussed using the mean-squared radius formula

$$
\left\langle r^{2}\right\rangle_{\beta} \approx\left(1+\frac{5}{4 \pi} \beta^{2}\right)\left\langle r^{2}\right\rangle_{\beta=0},
$$

carried over from other applications, such as the analysis of energy shifts in muonic atom data [12], where the nuclear density is also required. In Ref. [11], $\left\langle r^{2}\right\rangle_{\beta}$ was interpreted as the mean-squared radius of the deformed projectile deduced directly from cross section data using a spherical density OL reaction calculation. Equation (1) was then used to subtract the effects of the projectile deformation through a chain of isotopes to yield a spherical part of the nuclear radius $\left\langle r^{2}\right\rangle_{\beta=0}$. In [10] the onset of deformation, going from spherical to deformed Hartree-Fock calculations, was shown to lead to an increased rms size for the projectile, consistent with Eq. (1). This increased rms size then enhanced the cross section obtained using a spherical density OL calculation.

Within the reaction cross section calculation, the deformed density function enters the nuclear transparency (see below) in the argument of an exponential function. It is not clear therefore to what extent the cross section computed using a spherical angle average of the deformed density in this exponent will yield an accurate deduced matter radius. In this Brief Report we therefore calculate the reaction cross section, taking explicit account of the effects of the projectile (or target) deformation in the collision. We show that Eq. (1), interpreted appropriately, can be used to provide an accurate estimate of these effects.

The projectile nucleus, denoted (1), will be assumed to be quadrupole deformed with deformation $\beta \equiv \beta_{2}$. The orientation of the symmetry axis is denoted $\hat{\boldsymbol{\Omega}}$. The target nucleus, denoted (2), will be assumed to be spherical. We consider, for simplicity only, a zero-range underlying $N N$ interaction. Figure 1 shows the coordinates used in our model calculations where $\boldsymbol{R}$, the projectile-target separation, has cylindrical coordinates, $\boldsymbol{R} \equiv(\boldsymbol{b}, Z)$, with respect to the beam direction as the $Z$ axis. The optical limit reaction cross section for a fixed orientation of the incident projectile is then [9]

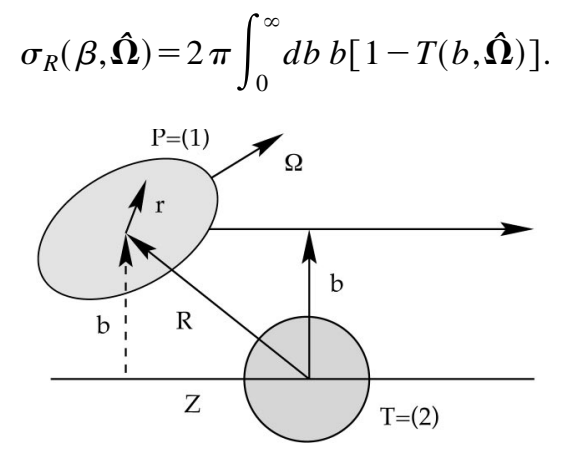

FIG. 1. Schematic representation of the collision of the deformed projectile and spherical target nuclei at an impact parameter $b$. The cylindrical coordinates used are shown. 
The transparency of the collision at center of mass (c.m.) impact parameter $b$ is

$$
T(b, \hat{\mathbf{\Omega}})=\exp \left[-\sigma_{N N} \mathcal{X}(b, \hat{\mathbf{\Omega}})\right],
$$

where $\sigma_{N N}$ is the isospin average of the free $n n$ and $n p$ cross sections appropriate for the specific projectile and target. The $Z$-integrated overlap of the projectile and target ground state densities, $\rho^{(i)} \quad(i=1,2)$, is

$$
\mathcal{X}(b, \hat{\mathbf{\Omega}})=\int_{-\infty}^{\infty} d Z \int d \boldsymbol{r} \boldsymbol{\rho}^{(1)}(\boldsymbol{r}, \hat{\mathbf{\Omega}}) \boldsymbol{\rho}^{(2)}(|\boldsymbol{R}+\boldsymbol{r}|) .
$$

We assume a Woods-Saxon form factor with a quadrupole-deformed radius parameter for the density of projectile nucleus (1), of mass $A_{1}$. Thus

$$
\begin{gathered}
\rho^{(1)}(\boldsymbol{r}, \hat{\boldsymbol{\Omega}})=\boldsymbol{\rho}_{0}^{(1)}(1+\exp \{[r-R(\hat{\boldsymbol{\Omega}})] / a\})^{-1}, \\
R(\hat{\mathbf{\Omega}})=R_{0}\left[1+\beta_{2} Y_{20}(\hat{\boldsymbol{\Omega}})\right],
\end{gathered}
$$

with multipole expansion

$$
\begin{aligned}
\rho^{(1)}(\boldsymbol{r}, \hat{\mathbf{\Omega}}) & =4 \pi \sum_{k q} \frac{\boldsymbol{\rho}_{k}^{(1)}(r)}{(2 k+1)} Y_{k q}(\hat{\boldsymbol{r}}) Y_{k q}^{*}(\hat{\mathbf{\Omega}}) \\
& =\sum_{k} \rho_{k}^{(1)}(r) P_{k}(\hat{\boldsymbol{r}} \cdot \hat{\mathbf{\Omega}}) .
\end{aligned}
$$

These multipole form factors $\rho_{k}^{(1)}(r)$ have been expressed analytically (to order $\beta_{2}^{3}$ ) by Fäldt and Glauber, Appendix B of Ref. [9]. The overall strength parameter $\rho_{0}^{(1)}$ and radial size parameter $R_{0}$ of the Woods-Saxon form factor are determined by the required volume integral and the projectile rms radius (assuming a fixed value for the diffuseness $a$ ), i.e.,

$$
A_{1}=4 \pi \int_{0}^{\infty} d r r^{2} \rho_{0}^{(1)}(r), \quad\left\langle r^{2}\right\rangle_{A_{1}}=\frac{4 \pi}{A_{1}} \int_{0}^{\infty} d r r^{4} \rho_{0}^{(1)}(r) .
$$

We assume a spherical Gaussian density for the target nucleus (2), of mass $A_{2}$,

$$
\rho^{(2)}(x)=\rho_{0}^{(2)} \exp \left(-\gamma x^{2}\right),
$$

with a strength $\rho_{0}^{(2)}$ and inverse range $\gamma$ determined by $A_{2}$ and the target rms radius,

$$
A_{2}=\rho_{0}^{(2)}(\pi / \gamma)^{3 / 2}, \quad\left\langle r^{2}\right\rangle_{A_{2}}=3 /(2 \gamma) .
$$

Upon substituting for $x^{2}=|\boldsymbol{R}+\boldsymbol{r}|^{2}$, then, in Eq. (4) we have

$$
\begin{aligned}
\rho^{(2)}(|\boldsymbol{R}+\boldsymbol{r}|)= & \rho_{0}^{(2)} \exp \left(-\gamma\left[R^{2}+r^{2}\right]\right) 4 \pi \\
& \times \sum_{k q} i^{k} j_{k}(2 i \gamma R r) Y_{k q}(\hat{\boldsymbol{R}}) Y_{k q}^{*}(\hat{\boldsymbol{r}}) .
\end{aligned}
$$

It follows, combining these results and the properties of the spherical harmonics, that the $Z$-integrated density overlap in Eq. (4) can be written

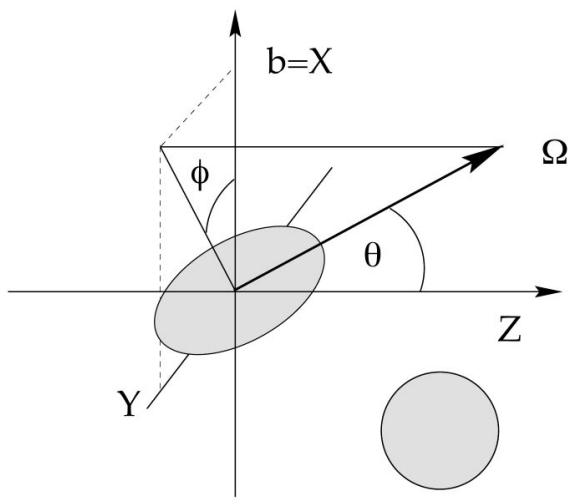

FIG. 2. Spherical polar coordinates used for the description of the orientation of the symmetry axis of the deformed projectile. The projectile c.m. and the target c.m. lie in the $X-Z$ plane.

$$
\mathcal{X}(b, \hat{\mathbf{\Omega}})=4 \pi \exp \left(-\gamma b^{2}\right) \sum_{k} \int_{-\infty}^{\infty} d Z \mathcal{O}_{k}(b, Z) P_{k}(\hat{\boldsymbol{R}} \cdot \hat{\boldsymbol{\Omega}})
$$

where, with $R=\sqrt{b^{2}+Z^{2}}$,

$$
\begin{aligned}
\mathcal{O}_{k}(b, Z)= & \rho_{0}^{(2)} \exp \left(-\gamma Z^{2}\right) \int_{0}^{\infty} d r r^{2} \rho_{k}^{(1)}(r) \\
& \times \exp \left(-\gamma r^{2}\right) i^{k} j_{k}(2 i \gamma R r) .
\end{aligned}
$$

If $\hat{\boldsymbol{\Omega}}$ is expressed using spherical polar angles $(\theta, \phi)$, with respect to the $Z$ axis in the projectile beam direction and $X$ axis along the c.m. impact parameter $\boldsymbol{b}$ (Fig. 2), then

$$
\hat{\boldsymbol{R}} \cdot \hat{\boldsymbol{\Omega}}=[b \sin \theta \cos \phi+Z \cos \theta] / R .
$$

Assuming the projectile beam is unpolarized, we must average over all orientations $\hat{\boldsymbol{\Omega}}$, and

$$
\sigma_{R}(\beta)=\frac{1}{4 \pi} \int_{0}^{2 \pi} d \phi \int_{0}^{\pi} \sin \theta d \theta \quad \sigma_{R}(\beta, \hat{\mathbf{\Omega}}) .
$$

As typical, we consider the reaction cross section for ${ }^{17} \mathrm{~N}$ scattering from ${ }^{12} \mathrm{C}$ at a laboratory energy of $700 \mathrm{MeV} /$ nucleon. This system was studied in some detail by Kitagawa et al. [10] in the case of spherical density reaction calculations. At this energy, for a ${ }^{12} \mathrm{C}$ target, the isospinaveraged $N N$ cross section is $\sigma_{N N}=4.087 \mathrm{fm}^{2}$, based on experimental data [10]. We assume a spherical Gaussian density for the ${ }^{12} \mathrm{C}$ target with a point nucleon rms matter radius of $2.32 \mathrm{fm}$, consistent with the charge radius deduced from electron scattering. For the projectile density we assume a Woods-Saxon form factor with a quadrupoledeformed radius. Multipoles with $k=0,2$, and 4 are included in Eq. (11). The diffuseness parameter is kept fixed at the value $a=0.564 \mathrm{fm}$ throughout.

We first consider the density for ${ }^{17} \mathrm{~N}$ from the spherical Hartree-Fock calculation. This yields a calculated ${ }^{17} \mathrm{~N} \mathrm{rms}$ matter radius of $2.682 \mathrm{fm}$, taken from Table 1 of Ref. [10]. Assuming a spherical Woods-Saxon form factor for this density, with volume integral $A_{1}=17$ and rms radius $2.682 \mathrm{fm}$, we obtain the parameters 


$$
\rho_{0}^{(1)}=0.240103 \mathrm{fm}^{-3}, \quad R_{0}=2.16121 \mathrm{fm}, \quad \beta_{2}=0 .
$$

The calculated (spherical) optical limit reaction cross section is $\sigma_{R}(0)=1065.7 \mathrm{mb}$.

The deformed Hartree-Fock calculations of [10] give a prolate deformation parameter of $\delta=+0.218$, corresponding to a $\beta \equiv \beta_{2}=0.2289$. For comparison with the spherical case above, and to clarify the effect of deformation, we now assume a quadrupole-deformed Woods-Saxon density with this $\beta_{2}$, but with the same rms matter radius as for the spherical case $(2.682 \mathrm{fm})$. The required Woods-Saxon density parameters are

$\rho_{0}^{(1)}=0.249395 \mathrm{fm}^{-3}, \quad R_{0}=2.10776 \mathrm{fm}, \quad \beta_{2}=0.2289$.

The deformed projectile OL reaction cross section, after integrating over all orientations, is now $\sigma_{R}(\beta)=1057.6 \mathrm{mb}$. We note that treating explicitly this deformed structure leads to a reduction of $0.8 \%$ in the calculated cross section compared to using the OL calculation for a spherical projectile with the same rms matter radius.

This difference is understood by considering the calculated reaction cross sections for several fixed orientations of the projectile. With the polar coordinates of Fig. 2 and the parameters of Eq. (15), the reaction cross sections $\sigma_{R}(\beta, \hat{\mathbf{\Omega}})$ for the following fixed $\hat{\mathbf{\Omega}} \equiv(\theta, \phi)$ orientations are

$$
\begin{gathered}
\sigma_{R}(\beta, 0,0)=1023.4 \mathrm{mb}, \\
\sigma_{R}\left(\beta, \frac{\pi}{2}, 0\right)=1141.9 \mathrm{mb}, \\
\sigma_{R}\left(\beta, \frac{\pi}{2}, \frac{\pi}{2}\right)=1006.3 \mathrm{mb} .
\end{gathered}
$$

The largest and smallest cross sections occur for $\theta=\pi / 2$ and $\phi=0, \pi / 2$, respectively. The calculations at these different angular extremes represent very significant changes, of $+7.2 \%$ to $-5.6 \%$, about the spherical result. The orientation averaging results in only a $0.8 \%$ reduction in the calculated cross section compared to the spherical OL calculation, reflecting the fact that the larger cross sections are encountered in relatively fewer orientations of the elipsoid. Were the projectile produced with any significant degree of alignment, the results above show that the implications for deduced sizes could be significant. Use of an oblate deformation $\beta_{2}=$ -0.2289 requires, for the same rms radius, the parameters

$$
\begin{gathered}
\rho_{0}^{(1)}=0.247895 \mathrm{fm}^{-3}, \quad R_{0}=2.11432 \mathrm{fm}, \\
\beta_{2}=-0.2289 .
\end{gathered}
$$

The angle-averaged cross section is $\sigma_{R}(\beta)=1058.6 \mathrm{mb}$, showing a high degree of symmetry with respect to the sign of $\beta_{2}$.
In practice, the OL reaction theory is used to extract a value for the rms size of the projectile nucleus by fitting a given experimental cross section datum, rather than the rms radius being known. The question is therefore, how do the radii deduced by fitting a given cross section value differ when using the spherical and deformed versions of the reaction theory? By varying the rms matter radius of a spherical Woods-Saxon density we are able to find an effective spherical density that reproduces the same reaction cross section as the deformed case. The required spherical density has a rms radius of $2.654 \mathrm{fm}$, which calculates a $\sigma_{R}(0)=1057.6 \mathrm{mb}$, and has parameters

$$
\rho_{0}^{(1)}=0.254776 \mathrm{fm}^{-3}, \quad R_{0}=2.10304 \mathrm{fm}, \quad \beta_{2}=0 .
$$

So a spherical density with rms radius $2.654 \mathrm{fm}$ generates the same OL reaction cross section as does a deformed density, with $\beta_{2}=0.2289$ and larger rms radius $2.682 \mathrm{fm}$, in a deformed OL calculation. This difference in these deduced rms radii is a $1.06 \%$ effect. The estimate of this effect based on Eq. (1),

$$
\left\langle r^{2}\right\rangle_{\beta} \approx\left(1+\frac{5}{4 \pi} \beta^{2}\right)\left\langle r^{2}\right\rangle_{\beta=0},
$$

is $1.04 \%$, in reasonable agreement with our calculated value. Whereas a naive application of this equation to rms radii used in spherical calculations will lead to the deformation driving larger cross sections, this is not the case in the deformed reaction calculations reported here.

The example presented here clarifies the manner in which Eq. (1) should be interpreted in the case of root mean square radius deductions for deformed projectiles. Our explicit treatment of the deformed projectile density is shown, for an unpolarized projectile beam, to lead to smaller calculated OL cross sections than are obtained for a spherical density with the same rms radius, i.e., $\sigma_{R}(\beta)<\sigma_{R}(0)$. It follows that a fit made to cross section data using a spherical density OL calculation will derive a $\left\langle r^{2}\right\rangle_{\beta=0}^{1 / 2}$ which will underestimate the actual projectile rms size. We have shown, however, that the prescription given by Eq. (1) accounts reasonably for this difference, for physical values of $\beta$. It can be used to adjust the rms radius $\left\langle r^{2}\right\rangle_{\beta=0}^{1 / 2}$ extracted from a given experimental cross section using $\sigma_{R}(0)$ to the required value $\left\langle r^{2}\right\rangle_{\beta}^{1 / 2}$ which would be obtained if one had analyzed the same cross section using $\sigma_{R}(\beta)$. This example also confirms that, overall, the deformation effects on deduced cross sections are relatively small, compared, for instance, with the few-body effects discussed in our introductory comments and in Refs. [6-8]. Were the projectiles produced with any significant degree of alignment, our calculations show that the implications for calculated cross sections and deduced sizes could be much more significant.

The financial support of the United Kingdom Engineering and Physical Sciences Research Council (EPSRC) in the form of Grant No. GR/J95867 is gratefully acknowledged. 
[1] R.J. Glauber, in Lectures in Theoretical Physics, edited by W.E. Brittin (Interscience, New York, 1959) Vol. 1, p. 315.

[2] W. Czyz and L.C. Maximon, Ann. Phys. (N.Y.) 52, 59 (1969).

[3] P.J. Karol, Phys. Rev. C 11, 1203 (1975).

[4] I. Tanihata et al., Phys. Rev. Lett. 55, 2676 (1985); Phys. Lett. 160B, 380 (1985).

[5] I. Tanihata, T. Kobayashi, O. Yamakawa, S. Shimoura, K. Ekuni, K. Sugimoto, N. Takahashi, T. Shimoda, and H. Sato, Phys. Lett. B 206, 592 (1988).

[6] J.S. Al-Khalili and J.A. Tostevin, Phys. Rev. Lett. 76, 3903 (1996); J.S. Al-Khalili, J.A. Tostevin, and I.J. Thompson,
Phys. Rev. C 54, 1843 (1996).

[7] J.A. Tostevin, R.C. Johnson, and J.S. Al-Khalili, Nucl. Phys. A630, 340c (1998).

[8] J.A. Tostevin and J.S. Al-Khalili, Phys. Rev. C 59, R5 (1999).

[9] G. Fäldt and R.J. Glauber, Phys. Rev. C 42, 395 (1990).

[10] H. Kitagawa, N. Tajima, and H. Sagawa, Z. Phys. A 358, 381 (1997).

[11] T. Suzuki et al., Nucl. Phys. A630, 661 (1998).

[12] G. Fricke, J. Herberz, Th. Hennemann, G. Mallot, L.A. Schaller, L. Schellenberg, C. Pillar, and R. Jacob-Guillarmod, Phys. Rev. C 45, 80 (1992). 